\title{
Gender Differences in Authorship of Clinical Problem-Solving Articles
}

\author{
Elizabeth Adler, MD ${ }^{1 *}$, Andrew Hobbs, MA², Gurpreet Dhaliwal, MD¹,3, Jennifer M Babik, MD, PhD ${ }^{1}$
}

${ }^{1}$ Department of Medicine, University of California, San Francisco, California; ${ }^{2}$ Department of Economics, University of San Francisco, California; ${ }^{3}$ Medical Service, San Francisco VA Medical Center, San Francisco, California.

Authors of clinical reasoning exercises analyze diagnostic dilemmas and serve as role models of clinical excellence. We investigated the percentage of women authors in the clinical problem-solving series of three general medicine journals from the inaugural article in each series until July 2019. Women were underrepresented among first, last, and all authors. While the percentage of women among first and all authors has increased, women still constituted $<40 \%$ of all authors and $\leq 25 \%$ of last authors, and there have been no significant increases in women last authors in any of the three journals. Including more women in clinical reasoning exercises is an opportunity to amplify the voices of women as master clinicians. Journal of Hospital Medicine 2020;15:475-478. (C) 2020 Society of Hospital Medicine large body of evidence has demonstrated significant gender disparities in academic medicine. Women are less likely than men to reach the rank of full professor, be speakers at Grand Rounds, and author studies in medical journals. ${ }^{1-4}$ Gender-based differences in these achievements reduce the visibility of women role models in all academic medicine domains, including research, education, health systems leadership, and clinical excellence. Clinical problem-solving exercises are an opportunity to highlight the skills of women physicians as master clinicians and to establish women as clinician role models.

Clinical problem-solving exercises are highly visible demonstrations of clinical excellence in the medical literature. These exercises follow a specific format in which a clinician analyzes a diagnostic dilemma in a step-by-step manner in response to sequential segments of clinical data. The clinical problemsolving format was introduced in 1992 in the New England Journal of Medicine and has been adopted by other journals. ${ }^{5}$ (The clinical problem-solving format differs from the clinical pathologic conference format, in which an entire case is presented followed by an extended analysis). Clinical problemsolving publications are forums for learners of all levels to witness an expert clinician reason through a case.

Authorship teams on clinical reasoning exercises typically include the patient's physician(s), specialists relevant to the final diagnosis, and the invited discussant who analyzes the clinical dilemma. Journals stipulate in the author instructions, series introductions, or standardized manuscript text of the series that the discussant be a skilled and experienced clini-

*Corresponding Author: Elizabeth Adler, MD; Email: elizabeth.c.adler@gmail. com; Telephone: 650-302-2949; Twitter: @eadlermd.

Published online first July 22, 2020.

Find additional supporting information in the online version of this article.

Received: January 30, 2020; Revised: May 4, 2020; Accepted: May 7, 2020

๔ 2020 Society of Hospital Medicine DOI 10.12788/jhm.3465 cian. ${ }^{5,6}$ The patient's physicians who initiate the clinical reasoning manuscript typically select the discussant; in some journals, the series editors may provide input on discussant choice. To our knowledge, this is the only author role in the medical literature in which authors are invited specifically for their diagnostic reasoning ability.

While women have been authors on fewer original research articles and guest editorials than men have, ${ }^{3}$ the proportion of women among authors of published clinical reasoning exercises is unknown. This represents a gap in our understanding of the landscape of gender inequity in academic medicine. We sought to determine the proportion of women authors in major clinical problem-solving series and examine the change in women authorship over time.

\section{METHODS}

We selected published clinical problem-solving series targeting a general medicine audience. We excluded general medicine journals in which authors were restricted to one institution or those in which the clinical problem-solving format was not a regular series. Series which met these criteria were the Clinical Problem-Solving series in the New England Journal of Medicine (NEJM), the Clinical Care Conundrums series in the Journal of Hospital Medicine (JHM), and the Exercises in Clinical Reasoning series in the Journal of General Internal Medicine (JGIM). We analyzed the proportion of women authors in each clinical reasoning series from the inaugural articles (1992 for NEJM, 2006 for JHM, and 2010 for JGIM) until July 2019. We also analyzed the change in proportion of women authors from year to year by using data up to 2018 to avoid including a partial year.

We used the gender-guesser python library ${ }^{7}$ to categorize the gender of first, last, and all authors based on their first names. The library uses a database of approximately 40,000 names ${ }^{8}$ and maps first names to the genders they are associated with across languages, classifying each name as "man," "woman," "mostly man," "mostly woman," "androgynous," or "unknown." When a 
TABLE. Number of Total Articles, Total Authors, and Women Among First, Last, and All Authors ${ }^{a}$

\begin{tabular}{|c|c|c|c|c|c|}
\hline & Total number of articles & Total number of authors & No. Women First Authors (\%) & No. Women Last Authors (\%) & No. Women Among All Authors (\%) \\
\hline 1992 & 12 & 17 & $0(0.0)$ & $0(0.0)$ & $0(0.0)$ \\
\hline Total $^{b}$ & 280 & 1,026 & $80(28.6)$ & $28(10.0)$ & $224(21.8)$ \\
\hline \multicolumn{6}{|l|}{ JHM } \\
\hline Total $^{b}$ & 83 & 362 & $36(43.4)$ & $6(7.2)$ & $83(22.9)$ \\
\hline \multicolumn{6}{|l|}{ JGIM } \\
\hline 2010 & 2 & 9 & $0(0.0)$ & $2(100.0)$ & $2(22.2)$ \\
\hline 2018 & 4 & 18 & $2(50.0)$ & $1(25.0)$ & $7(38.9)$ \\
\hline
\end{tabular}

name is commonly associated with multiple genders, or is associated with different genders in different languages, it is classified either as mostly man, mostly woman, or androgynous. When a name is not found in the database, it is classified as unknown. For all names classified by the database as unknown, androgynous, or mostly man/mostly woman, we determined gender identities by finding the authors' institutional webpages and consulting their listed gender pronouns. We used gender based on first name to best approximate what a reader would interpret as the author's gender. We used gender rather than biological sex because authors may have changed their names to better express their gender identity, which may differ from sex assigned at birth.

To test for the statistical significance of changes in the proportion of women authors over time, we performed the Cochran-Armitage trend test. A $P$ value less than .05 was considered significant.

\section{RESULTS}

We analyzed 402 articles: 280 from NEJM, 83 from JHM, and 39 from JGIM. There were 1,026 authors of clinical reasoning articles from NEJM, 362 from JHM, and 168 from JGIM. The Table shows the number of total articles, total authors, and women among first, last, and all authors by journal and by year (inaugural year and 2018). Data for all years are shown in the Appendix Table.

Over the entire time period studied, the percentage of women across the three journals was lowest for last authors (28/280 [10.0\%] for NEJM, 6/83 [7.2\%] for JHM, and 9/39 [23.1\%] for JGIM) and highest for first authors (80/280 [28.6\%] for NEJM, 36/83 [43.4\%] for JHM, and 13/39 [33.3\%] for JGIM). The percentage of women among all authors was similar for all three journals: 224/1,026 (21.8\%) for NEJM, 83/362 (22.9\%) for JHM, and $36 / 168$ (21.4\%) for JGIM.

The Figure shows the change in percentage of women authors from year to year through 2018. There was a significant increase in the proportion of women first authors in NEJM (from 0/12 [0.0\%] in 1992 to 4/12 [33.3\%] in 2018; $P<.0001$ ) and JHM (from 2/5 [40.0\%] in 2006 to $7 / 9$ [77.8\%] in $2018 P=$ .01). There was also a significant increase in the proportion of women among all authors in NEJM (from 0/17 [0.0\%] in 1992 to $17 / 59$ [28.8\%] in 2018; $P<.0001$ ) and JHM (from 3/19 [15.8\%] in 2006 to $14 / 37$ [37.8\%] in 2018; $P=.005$ ). There was no significant change in the proportion of women last authors in any of the three journals. There were no statistically significant changes in JGIM authorship over time.

\section{DISCUSSION}

Clinical problem-solving exercises provide a forum for physicians to demonstrate diagnostic reasoning skills and clinical acumen. In this study, we focused on three prominent clinical problem-solving series in general medicine journals. We found that women authors were underrepresented in each series. The percentage of women authors has increased over time, especially among first and all authors; however, there was no change in the last author position. In all three series women still constituted less than $40 \%$ of all authors and less than $25 \%$ of last authors. In comparison, women currently constitute about $40 \%$ of general internal medicine physicians, and this proportion has been rapidly growing over time; women now represent over half of all medical school graduates as opposed to $6 \%$ in 1960.9 .10 Our findings are consistent with the large body of evidence that describes gender-based differences in opportunities within academic medicine.

Prior studies have shown that gender inequities in academic medicine stem from a longstanding culture of sexism; these inequities are perpetuated in part by having too few visible women role models and mentors. ${ }^{11}$ These factors may lead to editorial practices that favor articles written by men. In addition, women may be less likely to be invited as expert discussants if other authors have a bias of associating clinical exper- 


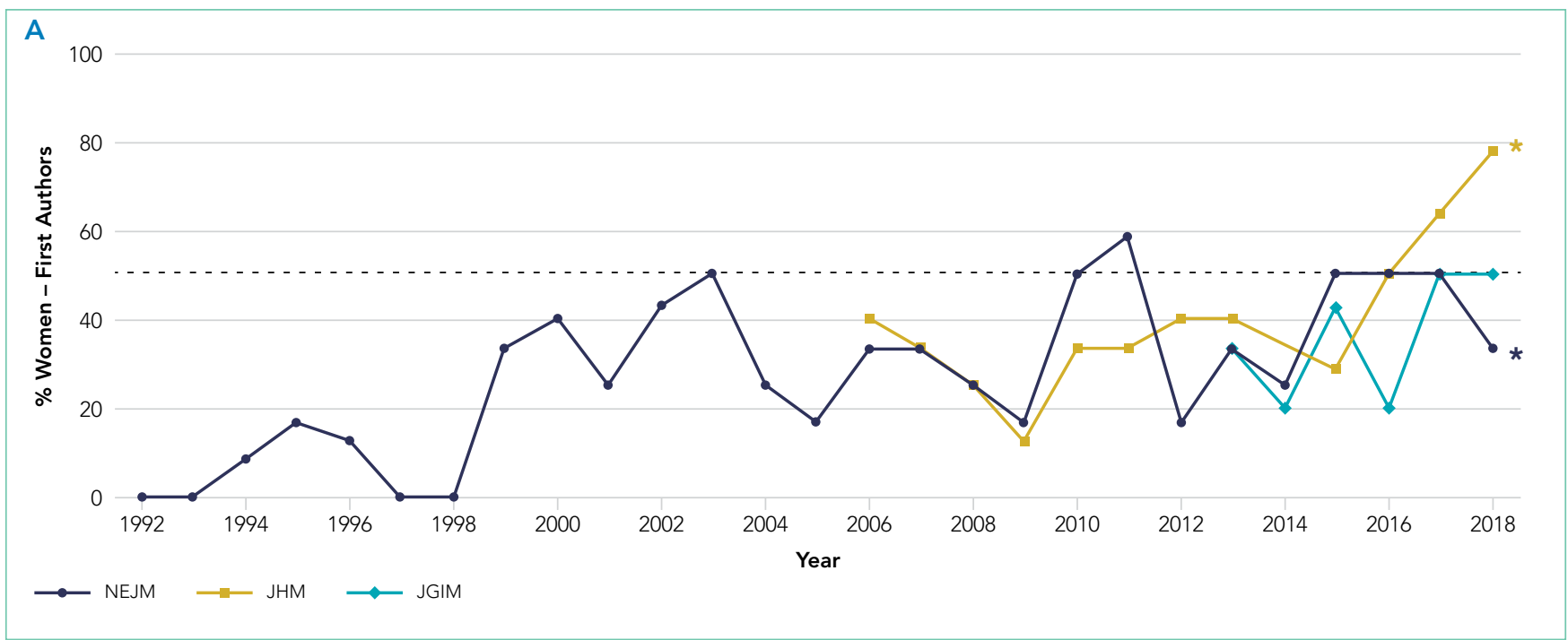

B

100

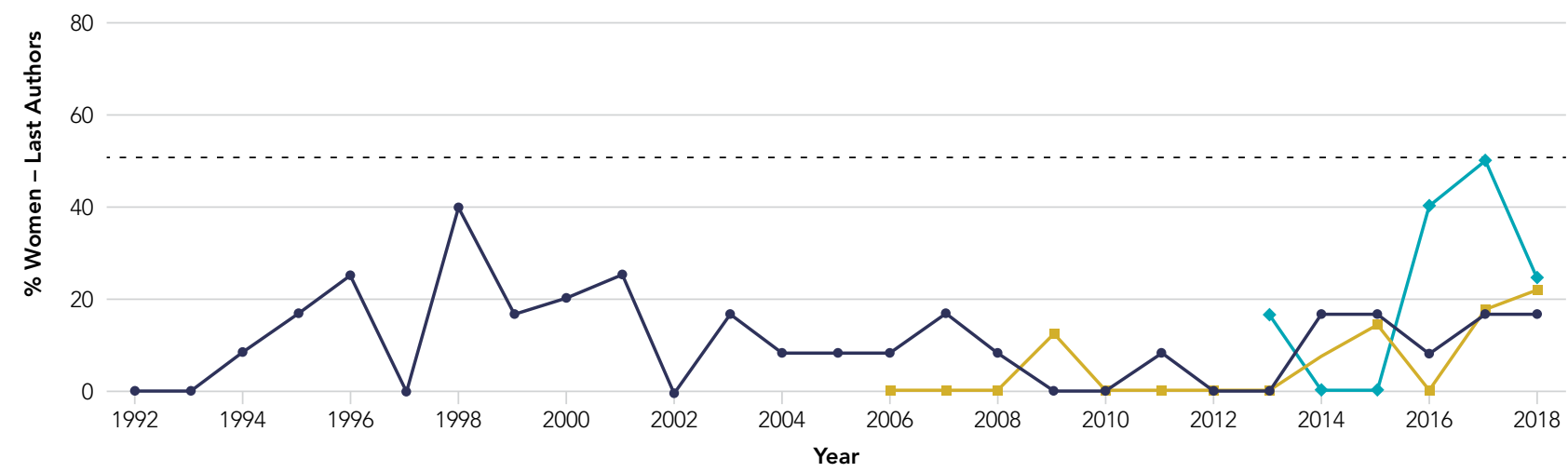

$\longrightarrow$ NEJM $\longrightarrow$ JHM $\multimap$ JGIM

C

100

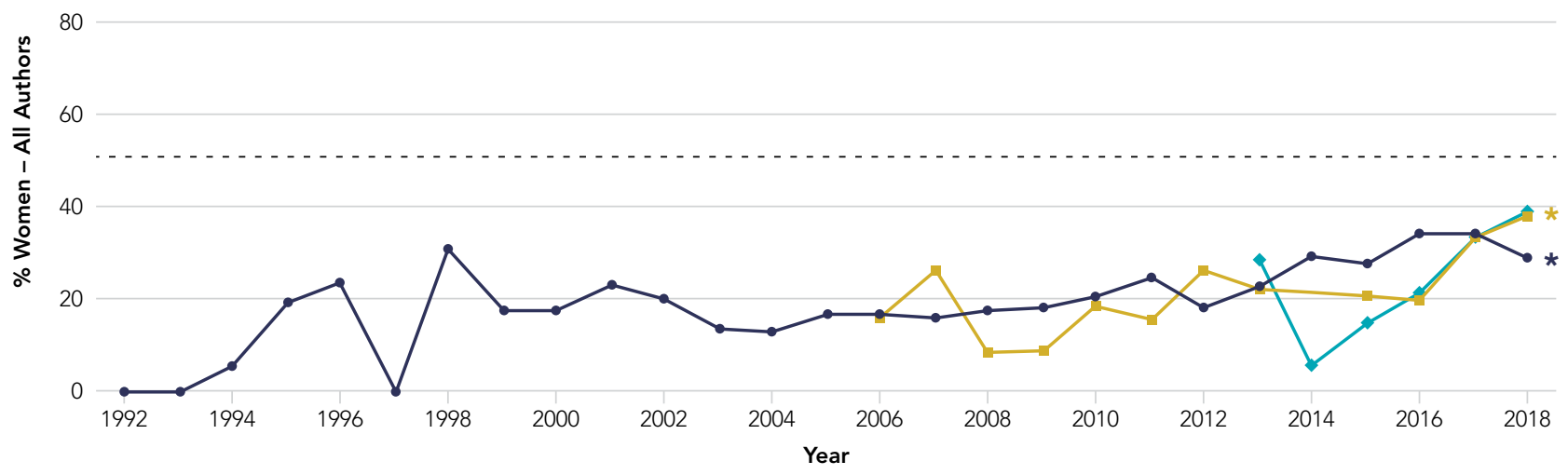

$\longrightarrow$ NEJM $\longrightarrow$ JHM $\longrightarrow$ JGIM

FIG. Percentage of Women Authors Over Time. Percentage of women among (A) first authors, (B) last authors, and (C) all authors for clinical reasoning exercises over time from inaugural article (1992 for the New England Journal of Medicine [NEJM], 2006 for the Journal of Hospital Medicine [JHM], 2010 for the Journal of General Internal Medicine [JGIM]) through the end of 2018. The asterisks following the line graphs of all and first authors for NEJM and JHM indicate statistically significant trends over time. The dotted line indicates 50\% women authors. Years with fewer than four published articles were excluded from the graph (JHM for 2014, JGIM from 2010-2012). 
tise with men physicians. This is consistent with data showing that women are less likely to be invited to write commentaries in peer-reviewed journals. ${ }^{12}$

Gender-based differences in authorship of clinical problem-solving publications also have important implications for women in medicine. In order to address the gender gap in academic achievement, women need visible role models and mentors. ${ }^{13}$ Including more women authors of clinical reasoning publications has the potential to establish more women as master clinicians and role models.

There are a number of actions that can help establish more women clinical problem-solving authors. Editorial boards and editors in chief should track their review and publication practices to hold themselves accountable to author diversity. For example, JHM has announced plans to analyze author representation of women and racial and ethnic minorities, including those among first and senior authors. ${ }^{14}$ Clinicians who are assembling author teams for clinical problem-solving manuscripts should also strongly consider if an equal number of men and women have been invited to serve as specialty consultants and case discussants.

Our study has limitations. We used a python library to classify author gender based on first name (supplemented by internet searches), which may have misclassified authors and did not take into account nonbinary gender identities. Because there is no convention for assigning the expert discussant to a specific author position, we could not determine the gender distribution of the discussants. However, given that women were underrepresented among first, last, and all authors in all three journals, they are likely a minority of discussants as well.

\section{CONCLUSION}

A preponderance of male voices in clinical reasoning exercises, in which learners see clinical role models, may perpetuate a culture in which women are not seen-and do not see themselves-as having the potential to be master clinicians. Including more women in clinical reasoning exercises is an op- portunity to amplify the voices of women as master clinicians and combat gender discrimination in medicine.

Disclosures: The authors report no conflicts of interest. Dr Dhaliwal is a US federal government employee and contributed as part of his official duties.

\section{References}

1. Jena $A B$, Khullar $D, H o O$, Olenski $A R$, Blumenthal $D M$. Sex differences in academic rank in US medical schools in 2014. JAMA. 2015;314(11):1149-1158. https://doi.org/10.1001/jama.2015.10680

2. Boiko JR, Anderson AJM, Gordon RA. Representation of women among academic grand rounds speakers. JAMA Intern Med. 2017;177(5):722-724. https://doi.org/10.1001/jamainternmed.2016.9646

3. Jagsi R, Guancial EA, Worobey CC, et al. The "gender gap" in authorship of academic medical literature--a 35-year perspective. N Engl J Med. 2006;355(3):281-287. https://doi.org/10.1056/nejmsa053910

4. González-Alvarez J. Author gender in The Lancet journals. Lancet. 2018;391(10140):2601. https://doi.org/10.1016/s0140-6736(18)31139-5

5. Kassirer JR. Clinical problem-solving - a new feature in the journal. N Engl J Med. 1992;326(1):60-61. https://doi.org/10.1056/nejm199201023260112

6. Henderson M, Keenan C, Kohlwes J, Dhaliwal G. Introducing exercises in clinical reasoning. J Gen Intern Med. 2010;25(1):9. https://doi.org/10.1007/ s11606-009-1185-4

7. Lead Ratings; 2019. Gender Guesser, Python 3. Accessed July 7, 2019. https://github.com/lead-ratings/gender-guesser

8. Michael J. genderReader. 2007. Accessed July 7, 2019. https://github.com/ cstuder/genderReader/blob/master/gender.c/gender.c

9. Association of American Medical Colleges. Active Physicians by Sex and Specialty, 2017. Physician Specialty Data Report. Accessed April 15, 2020. https://www.aamc.org/data-reports/workforce/interactive-data/active-physicians-sex-and-specialty-2017

10. Association of American Medical Colleges. More Women Than Men Enrolled in U.S. Medical Schools in 2017. AAMC Press Releases. December 17, 2017. Accessed April 15, 2020. https://www.aamc.org/news-insights/ press-releases/more-women-men-enrolled-us-medical-schools-2017

11. Yedidia MJ, Bickel J. Why aren't there more women leaders in academic medicine? the views of clinical department chairs. Acad Med. 2001;76(5):453465. https://doi.org/10.1097/00001888-200105000-00017

12. Thomas EG, Jayabalasingham B, Collins T, Geertzen J, Bui C, Dominici F. Gender disparities in invited commentary authorship in 2459 medical journals. JAMA Netw Open. 2019;2(10):e1913682. https://doi.org/10.1001/jamanetworkopen.2019.13682

13. Mullangi S, Jagsi R. Imposter syndrome: treat the cause, not the symptom. JAMA. 2019;322(5):403-404. https://doi.org/10.1001/jama.2019.9788

14. Shah SS, Shaughnessy EE, Spector ND. Leading by example: how medical journals can improve representation in academic medicine. J Hosp Med. 2019;14(7):393. https://doi.org/10.12788/jhm.3247 